\title{
On the disclination nature of pentagonal pyramids with high growth steps of electrolytic origin
}

\author{
N.N. Gryzunova ${ }^{\dagger}$ \\ †gryzunova-natalja@yandex.ru \\ Togliatti State University, 14 Belorusskaya St., Togliatti, 445667, Russia
}

\begin{abstract}
Functional materials based on base metals, which have a defect structure and developed surface are of a big scientific and practical interest due to their unusual physical and chemical properties. These materials can be used as catalytically active, sorbing and filtering elements, for example, in systems of catalytic waste water treatment or in a low-temperature catalysis of production of chemical agents. In this work, copper defective structures with a developed surface were produced by electrodeposition method with mechanical activation of the cathode and crystals growing on the cathode. Abrasive particles of $\mathrm{Al}$ and Si oxides moving in electrolyte were used as the activator. Such an activation leads to a formation of various crystal lattice defects including high-energy disclination type defects in crystals in the course of their growth. The activation promotes the formation of non-equilibrium, fragmented structures anda a developed surface of material. It was found that activation of the cathode allows for growing of pentagonal pyramids with high growth steps in large quantities. The paper presents the experimental results on a relaxation of internal elastic stress fields associated with disclination type defects in these pentagonal pyramids. In these pyramids, it is possible to observe the same channels of relaxation of internal elastic stress fields, which previously have been observed only in planar pentagonal microcrystals obtained at low overvoltage and without a mechanical activation of the cathode surface. This paper considers possible mechanisms of the formation of pentagonal pyramids, where one of the five sectors is missing, or splitting of the top of pyramids is observed.
\end{abstract}

Keywords: electrodeposition of copper, electrocrystallization with mechanical activation of the cathode, pentagonal pyramid.

\section{К вопросу о дисклинационной природе}

\section{пентагональных пирамид с высокими ступенями роста электролитического происхождения}

\author{
Грызунова H.H. ${ }^{\dagger}$ \\ Тольяттинский государственный университет, ул. Белорусская 14, Тольятти, 445667, Россия
}

Функциональные материалы на основе неблагородных металлов, обладающие дефектной структурой и развитой поверхностью, представляют большой научный и практический интерес благодаря своим необычным физико-химическим свойствам. Эти материалы могут быть использованы в качестве каталитически активных, сорбирующих и фильтрующих элементов, например, в системах каталитической очистки сточных вод или в низкотемпературном катализе при производстве химических веществ. В данной работе создание медных дефектных структур с развитой поверхностью осуществлялось методом электроосаждения с механической активацией катода и растущих на нем кристаллов. В качестве активатора применялись движущиеся в электролите абразивные частицы оксидов $\mathrm{Al}$ и $\mathrm{Si}$. Такая активация приводит к образованию в кристаллах, в процессе их роста, разнообразных дефектов кристаллического строения, в том числе, высокоэнергетических дефектов дисклинационного типа. Она способствует формированию неравновесных, фрагментированных структур и развитой поверхности материала. Обнаружено, что активация катода позволяет выращивать в большом количестве пентагональные пирамиды с высокими ступенями роста. В работе представлены экспериментальные результаты релаксации внутренних полей упругих напряжений, связанных с дефектами дисклинационного типа в таких пентагональных пирамидах. В них можно наблюдать такие же каналы релаксации внутренних полей упругих напряжений, какие ранее наблюдались лишь в плоских пентагональных микрокристаллах, полученных при низких перенапряжениях и без механической активации поверхности катода. В работе рассматривается возможный механизм образования пентагональных пирамид, у которых либо отсутствует один из пяти секторов, либо наблюдается расщепление вершины пирамиды.

Ключевые слова: электроосаждение меди, электрокристаллизация с механической активацией катода, пентагональные пирамиды. 


\section{1. Введение}

В последние годы опубликовано значительное число работ, посвященных методам получения, исследованию структуры и возможных физико-механических свойств частиц и кристаллов, имеющих оси симметрии пятого порядка. Такие пентагональные объекты выращены на основе не только чистых металлов, но и различных соединений [1]. В частности, в работах [2-5] получены пентагональные кристаллы меди [2], серебра [3], никеля [4] и других металлов [5]. В ряде работ [1,4,5] такие микрообъекты скорее являются исключением, чем правилом. В них авторы наблюдают одиночные кристаллы, которые образуются в узком интервале технологических параметров. Есть публикации и обзорные статьи $[2,3,6]$, в которых представлены технологические режимы электролиза для массового получения микрокристаллов с пентагональной симметрией. Однако, несмотря на большую проведенную работу, пока не созданы способы стабильно массового выращивания этих объектов, так как незначительные отклонения условий электролиза (флуктуации температуры, плотности тока или перенапряжения на катоде и т. д.) приводят к совершенно различающимся результатам.

В работах $[7,8]$ для стабильного получения высокой концентрации кристаллов меди микронных размеров в виде пентагональных пирамид с высокими ступенями роста и покрытий из них был найден и реализован новый подход. Он заключается в механическом воздействии на поверхность катода движущихся частиц абразива во время электроосаждения. Особенностью такого подхода является то, что активация катода осуществляется лишь на начальных этапах электрокристаллизации меди. Медные покрытия, полученные таким способом, состоят на $70-80 \%$ из пентагональных пирамид и могут быть использованы в низкотемпературном катализе [9]. Однако для прогнозирования возможностей предлагаемого метода необходимы не только детальные исследования влияния активирования на сам процесс образования и роста пентагональных пирамид, но и теоретическое обоснование, а также разработка и экспериментальные подтверждения возможных механизмов образования кристаллов с пентагональной симметрией в процессе электролиза с активацией катода.

Ранее в работах $[2,3,5,6,10-21]$ уже были сделаны попытки объяснить наличие пентагональной симметрии в частицах и кристаллах, имеющих микронные размеры. Теоретически было показано [10], исходя из энергетических соображений, что частицы с пентагональной симметрией устойчивы лишь до некоторого критического значения (порядка 100 нм). Практика показала [2,3,6-9,17-19], что пентагональная симметрия сохраняется при размерах кристаллов и частиц во много раз превышающих критические размеры. Многообразие пентагональных кристаллов микронных размеров, полученных в медных покрытиях, объяснялось наличием в них частичных семиградусных дисклинаций [6], а в работе [12] была теоретически обоснована эффективность применения дисклинационного подхода для анализа неоднородной упругой деформации, возникающей в пентагональных малых частицах и кристаллах. Дисклинационный подход использовался и для объяснения механизмов роста нитевидных микрокристаллов, призм и пирамид с пентагональной симметрией [13-15]. Факт роста кристаллов до размеров в десятки микрометров теоретически объяснялся релаксацией полей упругих напряжений, связанных с дефектами дисклинационного типа в кристаллах [16]. В некоторых работах [2, 17 - 19] показаны экспериментально наблюдаемые каналы релаксации полей упругих напряжений в плоских пентагональных микрокристаллах меди и серебра, доказана неразрывная связь пентагональной симметрии с дисклинациями. Однако, экспериментально наблюдаемые каналы релаксации полей упругих напряжений в пентагональных пирамидах с высокими ступенями роста, полученных в процессе электролиза под действием активатора, до сих пор в работах не были представлены.

Целью настоящей работы является демонстрация экспериментальных данных, подтверждающих дисклинационную природу пентагональных пирамид с высокими ступенями роста (рис. 1a), которые получаются в массовом количестве во время электроосаждения при высоких перенапряжениях и условии применения (на начальном этапе электрокристаллизации) механической активации поверхности катода и растущих на нем кристаллов. Также предлагается возможный механизм образования пентагональных пирамид, у которых либо отсутствует один из пяти секторов, либо наблюдается расщепление вершины пирамиды.

\section{2. Методика получения и исследования}

В работе пентагональные пирамиды меди с высокими ступенями роста выращивались методом электроосаждения на микросетках из нержавеющей стали марки $12 \mathrm{X} 18 \mathrm{H} 10$ с диаметром проволоки $55 \pm 3$ мкм и размером ячеек $90 \pm 4$ мкм из водного раствора $25 \%$ мас. сульфата меди с добавлением 5\% мас. серной кислоты. Электроосаждение велось с применением механической активации поверхности катода и растущих на нем кристаллов меди в потенциостатическом режиме при перенапряжениях 160 - 180 мВ в течение 10 - 15 минут и температуре электролита от 20 до $25^{\circ} \mathrm{C}$. Для этого применялась специально изготовленная электролитическая ячейка, подключенная к потенциостату P-150J фирмы Elins. Активирование катода осуществлялось на начальном этапе электрокристаллизации движущимися в электролите абразивными частицами оксидов алюминия и кремния $[8,20]$, взятыми примерно в соотношении 1:1. Частицы абразива-активатора предварительно обезжиривались, промывались, просушивались и добавлялись в элек-

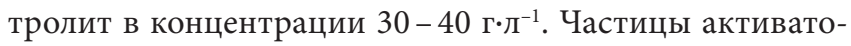
ра приводились в электролите в движение при помощи якоря магнитной мешалки, вращающегося со скоростью $60-70$ об/с.

Для проведения структурных исследований мест роста пентагональных пирамид осуществлялась электрополировка медной пленки, состоящей из пентагональных кристаллов, в $60 \%$ ортофосфорной кислоте в течение примерно 3 минут. 
Морфология поверхности кристаллов исследовалась на сканирующем электронном микроскопе (СЭМ) Carl Zeiss Sigma.

\section{3. Результаты и их обсуждение}

Детальные электронно-микроскопические исследования морфологии поверхности медных пентагональных пирамид с высокими ступенями роста (рис. 1), полученных в процессе электроосаждения с механической активацией катода, позволили обнаружить экспериментальные подтверждения их дисклинационной природы. В частности, замечено, что в покрытии одновременно можно наблюдать как «совершенные» пентагональные пирамиды (рис. 1a), так и пирамидальные пентагональные кристаллы, у которых отсутствует либо стык отдельных секторов по двойниковой границе, либо один из пяти секторов, составляющих пентагональный микрокристалл (рис. 1b). Стоит также отметить, что в морфологии пентагональных пирамид с высокими ступенями роста можно наблюдать расщепление вершины пирамиды (рис. 1с).

При этом замечено, что «совершенные» пентагональные пирамиды (рис. 1а) в радиальном направлении в области их основания в среднем не превышают размеров порядка 10 мкм. Другие пирамиды, у которых наблюдаются несовершенства в морфологии (открытый сектор, расщепление вершины), как правило, имеют средние размеры, превышающие 10 мкм.
Полученные экспериментальные факты можно трактовать как результат релаксации полей упругих напряжений, связанных с дефектами дисклинационного типа в них. В работах [2,6,12-19] отмечено, что основным атрибутом пентагональной симметрии является наличие дисклинации в кристаллах. В работах $[11,20]$ были представлены экспериментальные данные, позволяющие предположить, что пентагональные пирамиды с высокими ступенями роста, выращенные с применением механической активации катода, содержат в центре частичную семиградусную дисклинацию $\left(\omega=7^{\circ} 20^{\prime}\right)$ и пять сходящихся на ней двойниковых границ $[11,20]$. Тогда при дальнейшем росте пентагональной пирамиды в процессе электролиза упругая энергия в кристалле, связанная с дисклинацией, растет $\left(E \sim \omega^{2} R^{2}\right.$, где $R$ - радиус основания пирамиды) [12]. Поэтому устойчивость пентагональной симметрии в пирамидах, размеры которых превышают 10 мкм, может быть связана только с образованием в них дополнительных дефектов компенсирующих внутренние напряжения от дисклинации. В работе [12] показано, что упругая энергия в пентагональном кристалле может быть ослаблена за счет возникновения клиновидной области, образованной системой двойниковых границ. Это предполагает формирование частичной отрицательной клиновой дисклинации с углом клина $\omega^{1}$, соединенной с поверхностью кристалла системой из плоских дефектов в одном из тетраэдров (рис. $1 \mathrm{~b}$ и $2 \mathrm{a}, \mathrm{b})$. В результате этого можно наблюдать пентагональные пирамиды, у которых отсутствует один из секторов (рис. $1 \mathrm{~b}$ и $2 \mathrm{a}$ ).

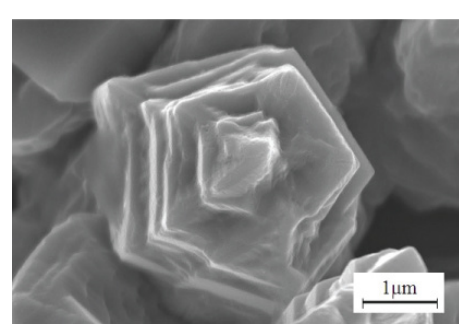

a

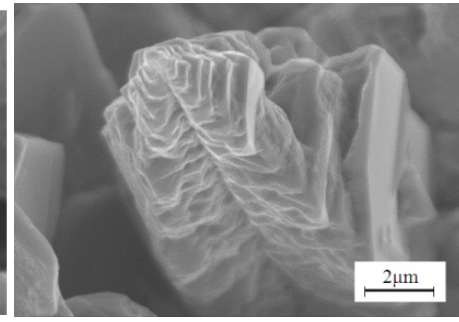

b

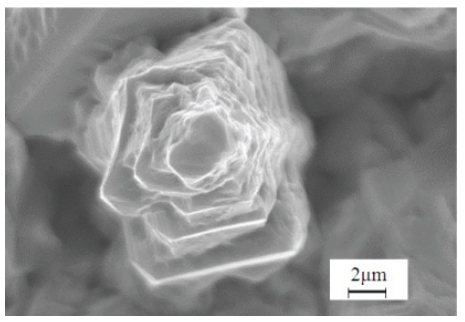

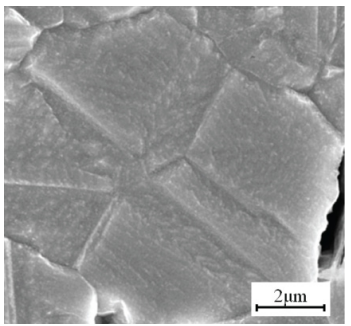

d

Рис.. 1. Микрофотографии пентагональных пирамид меди, полученных электролитическим способом с механической активацией катода и мест их роста: пентагональная пирамида с высокими ступенями роста (a); раскрытие сектора (b); расщепление вершины пирамиды (c); СЭМ изображение места роста пентагональной пирамиды с двумя вершинами после электрополировки (d).

Fig. 1. Micrographs of pentagonal pyramids of copper produced electrolytically by a mechanical activation of the cathode and growth places: pentagonal pyramid with high growth steps (a), disclosure of a sector (b); splitting of the top of the pyramid (c); SEM image of a two-peak pentagonal pyramid growth place after electropolishing (d).

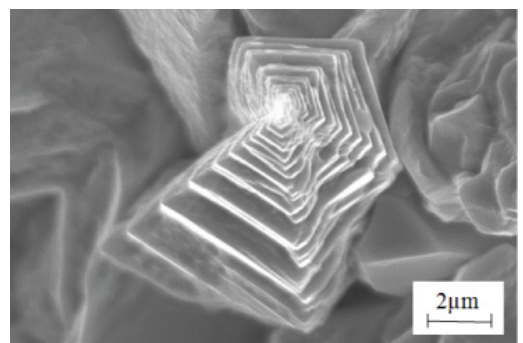

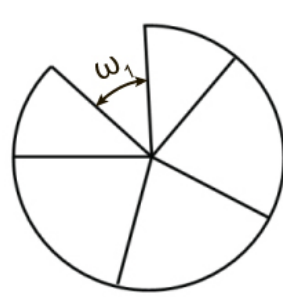

b

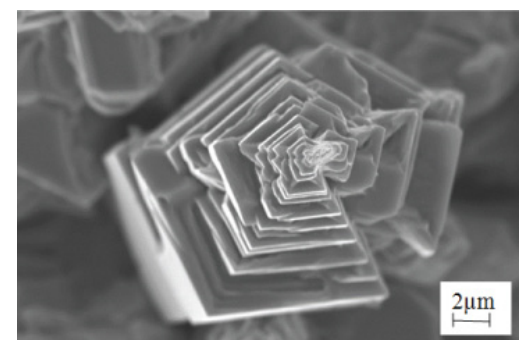



d

Рис. 2. Экспериментальные доказательства релаксации упругой энергии в пентагональных пирамидах, имеющих высокие ступени роста: отсутствие стыка секторов по двойниковой границе (СЭМ изображение и схема) (a, b); расщепление вершины пирамиды (СЭМ изображение и схема) (c, d).

Fig. 2. Experimental evidences for the relaxation of elastic energy in the pentagonal pyramids having high growth steps: lack of joint sectors on twin boundary (SEM image and schematic) (a, b); splitting of the pyramid apex (SEM image and diagram) (c, d). 
Наличие у пирамиды двух вершин (рис. 1с, и 2c) можно объяснить, опираясь на дисклинационные модели [12], следующим образом. При определенных условиях квадратичная зависимость энергии дисклинации от размера пирамиды вызывает энергетически благоприятное разложение частичной дисклинации $\left(\omega=7^{\circ} 20^{\prime}\right)$ на две меньшей мощности $\left(\omega^{2}>\omega_{1}^{2}+\omega_{2}^{2}\right)$ с излучением по одной из границ раздела $111<110>$ дислокаций. При этом происходит смещение оси частичной дисклинации, которое требует излучения дислокаций, что приводит к уменьшению длины границ двойников (рис. 1d, 2d). При расщеплении исходной дисклинации снижается энергия системы [22,23], поэтому пирамиды вырастают до размеров, существенно превышающих 10 мкм.

Для подтверждения теоретической модели (рис. 2d) образования двух вершин у пентагональных пирамид (рис. 2c) были проведены исследования микроструктуры основания пирамиды, которые показали наличие в пентагональном кристалле расщепления узла, где сходятся пять двойниковых границ (рис. 1d).

Учитывая все изложенное выше, можно предположить следующую последовательность развития процесса образования и роста пирамид во время электроосаждения с активатором.

Сначала на микросетке под действием движущихся в электролите абразивных частиц активатора формируются плоские пентагональные микрокристаллы меди, содержащие дефекты дисклинационного типа [11], возможные механизмы образования которых, были описаны ранее в работах $[2,6,15]$.

Затем при достижении плоскими микрокристаллами критических размеров (более 10 мкм), происходит реализация одного из возможных механизмов релаксации упругих напряжений $[2,18]$, в частности, раскрытие одного из секторов (рис. 2a,b) или расщепление узла дисклинации (рис. 1d, 2c,d). Далее уже на таком пентагональном микрокристалле происходит нарастание дискретным последовательным присоединением к кристаллу пентагональных слоев - “террас", растущих параллельно подложке, при этом формируются пентагональные пирамиды с высокими ступенями роста, у которых либо отсутствует один из секторов, либо имеются две вершины роста.

Эти экспериментальные факты хорошо согласуются с описанными ранее [13-15] механизмами образования пентагональных пирамид, которые вырастают на дефектах дисклинационного типа в подложках.

Исследование микроструктуры пирамид со стороны подложки подтвердило идею о том, что пирамиды растут из центра плоского пентагонального кристалла (рис. 1d).

\section{4. Заключение}

Таким образом, обнаруженные в экспериментах пути релаксации внутренних полей упругих напряжений указывают на дисклинационное происхождение пентагональных пирамид, которые выращены с применением механической активации поверхности катода и растущих на ней микрокристаллов меди. Эксперименты подтверждают нашу идею о том, что такие пирамиды формируются на плоских пентагональных кристаллах, первоначально образовавшихся на поверхности катода. Многообразие возможных путей релаксации упругой энергии, связанной с дисклинацией, позволяет пирамидам с высокими ступенями вырасти до достаточно больших размеров, сохраняя пентагональную симметрию.

Стоит также отметить, что основные каналы релаксации внутренних полей упругих напряжений ранее наблюдались лишь в пентагональных кристаллах других морфологических форм, полученных при низких перенапряжениях или токах и без механической активации катода $[1,14-16]$.

Благодарность/Acknowledgements. Исследование выполнено при финансовой поддержке РФФИ в рамках научного проекта № 16-02-00517 a. The reported study was funded by RFBR according to the research project № 16-02-00517 a.

\section{Литература/References}

1. K. Kitano, N. Maeda, T. Fukui et al. // Structure, 9 (6), 473 (2001).

2. A. A. Vikarchuk, A.P. Volenko, M. N. Tyurkov // Vestnik of Samara state technical University. Series: physics and mathematics sciences 27, 111 (2004) (in Russian) [А. А. Викарчук, А.П. Воленко, М.Н. Тюрьков, и др. // Вестник Самарского государственного технического университета. Серия: Физико-математические науки, 27, $111(2004)]$.

3. I.S. Yasnikov // Letters on Materials 1, 51 (2011) (in Russian) [И.С. Ясников // Письма о материалах, 1, 51 (2011)].

4. Golovin Y.I., Stolyarov R.A., Shuklin A. V. // Journal of technical physics 8, 105 (2013) (in Russian) [Головин Ю.И., Столяров Р.А., Шуклинов А.В. // Журнал технической физики, 8, 105 (2013)].

5. L. D. Marks and L. Peng // Journal of Physics: Condensed Matter 28, 48 (2016).

6. Vikarchuk A.A., Volenko A.P., Gamburg Yu.D. et. al., Russian Journal of Electrochemistry, 2, 207, (2004). (in Russian) [А. А. Викарчук, А. П. Воленко, Ю. Д. Гамбург, и др. // Электрохимия, 2, 207 (2004)].

7. Gryzunova N.N., Vikarchuk A.A., Bekin V.V., Romanov A.E. // Bulletin of the Russian Academy of Sciences: Physics. T. 79. №9, $1093-1097$ (2015) (in Russian) [Н.Н. Грызунова, А. А. Викарчук, В. В. Бекин, A. Е. Романов // Известия Российской академии наук. Серия физическая, том 79, №9, 1238 - 1243 (2015)].

8. Gryzunov A.M. // Vector of science of Togliatti state University 2 (36), 22 (2016) (in Russian) [А. М. Грызунов // Вектор науки Тольяттинского государственного университета, 2 (36), 22 (2016)].

9. Gryzunova N.N., Vikarchuk A.A., Gryzunov A.M., Maltsev A. V., Bekin V.V. // Fundamental nye problemy sovremennogo materialovedenia. T.13. № 4.444 (2016). (in Russian) [Грызунова Н.Н., Викарчук А.А., Грызунов А.М., Мальцев А.В., Бекин В.В. // Фундаментальные проблемы современного материаловедения. Т.13. № 4.444 (2016).] 
10. S. Ogawa, S. Ino // J. Cryst. Growth, 13/14, 48 (1972).

11. N.N. Gryzunova, A.A. Vikarchuk, M.N. Tyur'kov // Russian Metallurgy (Metally), 10, 924 (2016).

12. V.G. Gryaznov, A.M. Kaprelov, J. Heydenreich et. al., Crystal Research and Technology 34, 1091 (1999).

13. N.N. Gryzunova, A.A. Vikarchuk // Vector of science of Togliatti state University 4, 9 (2009) (in Russian) [Н.Н. Грызунова, А.А. Викарчук // Вектор науки Тольяттинского государственного университета, 4, 9 (2009)].

14. A. A. Vikarchuk, N. N. Gryzunova Materials Science, 6, 7 (2008) (in Russian) [А. А. Викарчук, Н. Н. Грызунова // Материаловедение, 6, 7 (2008)].

15. A. A. Vikarchuk, N. N. Gryzunova Materials Science, 5, 28 (2009) (in Russian) [А. А. Викарчук, Н. Н. Грызунова // Материаловедение, 5, 28 (2009)].

16. A.L. Kolesnikova, A.E. Romanov // Letters to journal of technical physics, 20, 73 (2007) (in Russian) [А. Л. Колесникова, А. Е. Романов // Письма в журнал технической физики, 20, 73 (2007)].

17. I. S. Yasnikov // Technical Physics. The Russian Journal of Applied Physics, 5, 133 (2007) (in Russian) [И. С. Ясников // Журнал технической физики, 5, 133 (2007)].

18. I. S. Yasnikov, A. A. Vikarchuk // Metal Science and Heat Treatment, 3, 13 (2007) (in Russian) [И.С. Ясников,
А. А. Викарчук // Металловедение и термическая обработка металлов, 3, 13 (2007)].

19. I. S. Yasnikov // Journal of Experimental and Theoretical Physics Letters (JETP Letters), 9-10, 592 (2013) (in Russian) [И.С. Ясников // Письма в Журнал экспериментальной и теоретической физики, 9-10, 592 (2013)].

20. Vikarchuk A.A., Gryzunova N.N., Gryzunov A.M., Romanov A.E. // Bulletin of the Tambov University. Series: Natural and technical Sciences T.21. № 3, 730 (2016) (in Russian) [Викарчук А.А., Грызунова Н.Н., Грызунов А. М., Романов А. Е. // Вестник Тамбовского университета. Серия: Естественные и технические науки Т.21. № 3, 730 (2016)].

21. A.G. Lipnitskii, D. N. Maradudin, D. N. Klimenko, et al.// Izvestiya Vysshikh Uchebnykh Zavedenii, Fizika. 52 (2). 27 (2009). (in Russian) [Липницкий А.Г., Марадудин Д.Н., Клименко Д.Н. и др. // Известия высших учебных заведений. Физика. 52 (2). 27 (2009)].

22. Romanov A.E., Vladimirov V.I. Disclinations in Crystalline Solids // Dislocations in Solids. Ed. F. R.N. Nabarro. Amsterdam, North-Holland, 9, 191 (1992).

23. R. De Witt Partial disclinations // Journal of Physics C: Solid State Physics, 5, 529 (1972) . 